\title{
Parameter Sensitivity Issues in Natural Field Orientation
}

\author{
G. Mirzaeva* and R.E. Betz \\ School of Electrical Engineering and Computer Science \\ University of Newcastle, NSW, Australia, 2308 \\ Email: *Galina.Mirzaeva@ newcastle.edu.au, ${ }^{\dagger}$ Robert.Betz@ newcastle.edu.au
}

\begin{abstract}
Natural Field Orientation (NFO) control was first developed in the mid 1980s and is a simplified version of the Stator Flux Oriented (SFO) control. NFO has some unique properties, including very high tolerance to the stator resistance errors. In this paper NFO's sensitivity to this and other parameter errors is explored, and its parameter robustness is compared to the polar form of SFO. A simple augmentation to both algorithms is developed which improves their parameter error robustness. This paper also shows that this augmentation, previously suggested to improve the NFO control frame stability, also has a positive effect on SFO frame stability by eliminating undesirable oscillations near the synchronous frequency.
\end{abstract}

\section{NOMENCLATURE}

$(x, y) \quad$ axes of the frame aligned with the actual position of the stator flux vector;

$(d, q) \quad$ control frame aligned with the estimated position of the stator flux vector;

$\underline{e}_{m s}, \underline{\hat{e}}_{m s}$ true and estimated stator flux voltage vectors;

$e_{s x}, e_{s y}$ stator flux voltage vector projections onto $(x, y)$ axes. Similarly for stator current vector: $i_{s x}$ and $i_{s y}$

$e_{s d}, e_{s q} \quad$ stator flux voltage vector projections onto $(d, q)$ axes. Similarly for stator current vector: $i_{s d}$ and $i_{s q}$;

$\underline{i}_{s \psi s} \quad$ the stator current space vector in a stator flux frame;

$\left|\underline{\psi}_{s}^{*}\right| \quad$ reference value of the stator flux magnitude. Similarly, ${ }^{*}$ denotes reference values for other quantities;

$\omega_{m s}, \hat{\omega}_{m s}$ true and estimated stator flux angular velocities, correspond to the angular velocities $(x, y)$ and $(d, q)$ frames;

$\theta_{m s}, \hat{\theta}_{m s}$ true and estimated stator flux angular positions;

$\theta_{e} \quad$ the error between the estimated and true stator flux frame angular positions - i.e. $\hat{\theta}_{m s}-\theta_{m s}$.

\section{INTRODUCTION}

Natural Field Orientation (NFO) is a "voltage model" [1] based stator flux vector control technique that is closely related to the better known Stator Flux Orientation (SFO). Voltage model based flux estimation has the following advantages over current model flux estimation techniques:

- The estimation of the flux is only dependent on knowledge of one machine parameter $\left(R_{s}\right)$ and a measurement of the applied stator voltage.

- The technique is inherently sensorless in that it does not require a measurement (or estimate) of the machine shaft speed for the estimator to work.

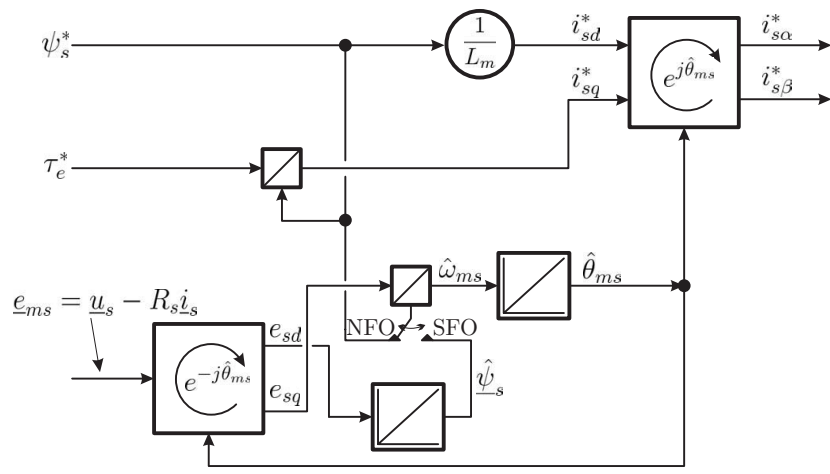

Fig. 1: Block diagram of the NFO and SFO algorithms.

The general form of SFO flux estimation is:

$$
\underline{\hat{\psi}}_{s}=\int_{t_{0}}^{t}\left(\underline{u}_{s}-R_{s} \underline{i}_{s}\right) d t+\underline{\hat{\psi}}_{s} \mid t_{0}
$$

where $\left.\underline{\psi}_{s}\right|_{t_{0}}$ is the estimated flux space vector value at time $t_{0}$.

Remark 1: One can see from (1) that $\hat{\psi}_{s}$ would be very sensitive to the initial condition, and the value of the stator resistance. If there is an error in $R_{s}$ then it will appear in an integrated form in $\underline{\hat{\psi}}_{s}$. Since the flux estimation itself is open loop, then other aspects of the control which are reliant on the flux estimate, and feedback via $\underline{e}_{m s}$, are the only means of preventing continuous integration of the error under steady state conditions. In addition to the sensitivity to $R_{s}$, pure integration techniques have difficulties with startup conditions. Depending on the initial values of voltage and current, and the precise details of the start-up transients, a DC offset can appear in the estimate. Again the lack of feedback in a pure integrator means that this offset will remain. This problem is usually addressed in practice by the use of a low pass filter approximation to the integrator.

NFO manages to avoid the issues cited above by a subtle, and to some degree non-obvious variation of the SFO algorithm. Instead of integrating the stator back-emf (i.e. $\underline{u}_{s}-R_{s} \underline{i}_{s}$ ) to get the flux, it simply assumes that the flux is at its reference value. Clearly if one assumes this, then the integration and its associated problems disappear.

Fig. 1 shows the essential difference between the NFO and polar form SFO algorithms. The switch selector in this figure determines whether the estimated angular velocity is derived from an estimated flux (in the case of SFO) or the 


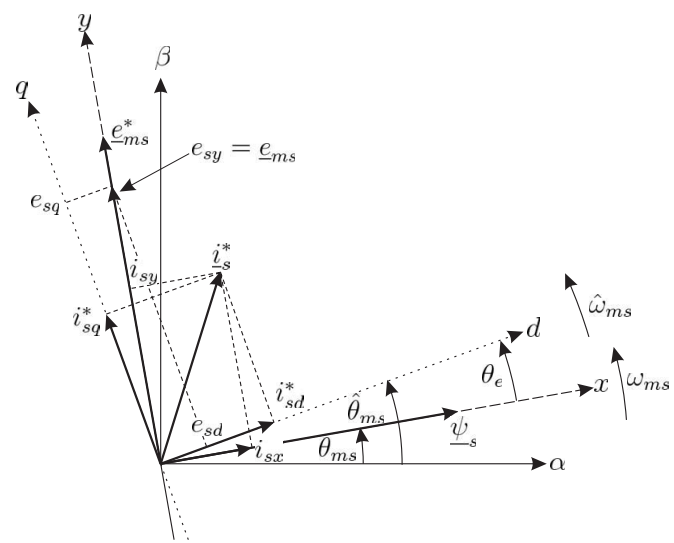

Fig. 2: Frame definitions for NFO under motoring.

flux reference with NFO. As can be seen from this figure, NFO and SFO are almost identical, except for the integration of $e_{s d}$ to get $\hat{\psi}_{s}$ in SFO, and the use of the flux reference, $\psi_{s}^{*}$, in NFO.

Remark 2: From a heuristic perspective it is not immediately obvious that the strategy of assuming that the flux is at its reference value will lead to a useful flux estimation strategy.

One of the interesting properties of NFO is that the $d q$ control frame has a tendency to align with the true frame when perturbed from the correct frame alignment position. This frame alignment property has been investigated in several previous papers by the authors [2]-[4], and will only be briefly discussed here.

Fig. 2 shows a snapshot of the frame positions under NFO and motoring. As can be seen in this figure, the $d q$ control frame is not aligned with the true $x y$ frame. It is assumed that the machine is current fed, therefore the reference currents $i_{s d}^{*}$ and $i_{s q}^{*}$ are impressed on the $d q$ axes. One can immediately see from this diagram that the misalignment means that the true flux producing current $i_{s x}$ is less than the desired current $i_{s d}^{*}$. Therefore $\left|\underline{\psi}_{s}\right|<\left|\underline{\psi}_{s}^{*}\right|$, which in turn means that $e_{s q}<e_{s y}$ and $e_{s y}=\left|\underline{e}_{m s}\right|$. Since under NFO the estimated frame angular velocity is determined through the equation [2], [5]-[7]:

$$
\hat{\omega}_{m s}=\frac{e_{s q}}{\left|\underline{\psi}_{s}^{*}\right|}=\frac{e_{s q}}{L_{m}\left|\underline{i}_{m s}^{*}\right|}
$$

whereas the $x y$ frame angular velocity is:

$$
\omega_{m s}=\frac{e_{s y}}{\left|\underline{\psi}_{s}\right|}=\frac{e_{s y}}{L_{m} i_{s y}}
$$

Since $e_{s q}<e_{s y}$ and $\left|\underline{\psi}_{s}^{*}\right|>\left|\underline{\psi}_{s}\right|$ then $\hat{\omega}_{m s}<\omega_{m s}$.

Remark 3: One can therefore conclude from this logic that if the frames become misaligned, then the geometry of the frames and their effect on the respective values of flux producing currents is such that the misalignment is statically stable and will tend to result in realignment of the frames.

Remark 4: The term static stability means that the above argument assumes that $d\left|\underline{\psi}_{s}\right| / d t=0$ - i.e. the machine is in steady state. In addition it does not take into account the cross-coupling dynamics of the machine. This is valid, since one can argue that if the frame movement is very slow then the dynamics of the machine become irrelevant. Under such a condition the tendency to realign will still remain.

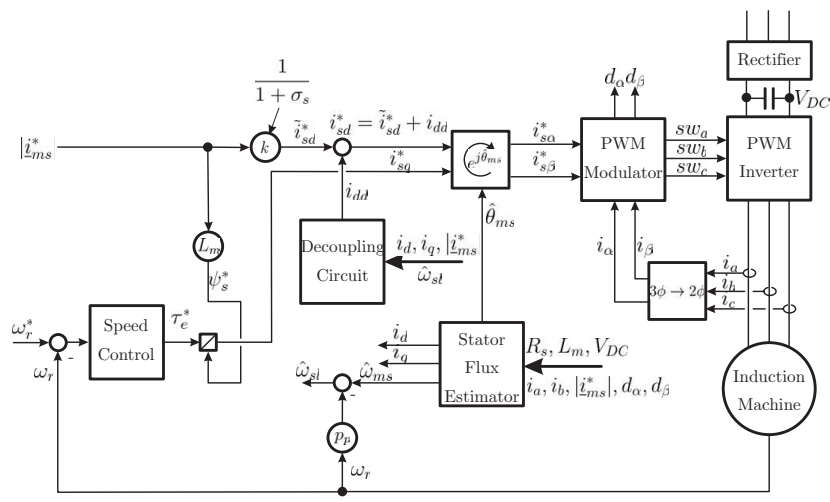

Fig. 3: Block diagram of experimental control system.

If one applies similar logic to the regeneration case that was used for motoring one is able to show that NFO is statically unstable over most of the regenerative region. This means that any frame error will tend to increase. A feedback solution to this problem based on using the fact that $e_{s d} \neq 0$ under misalignment conditions has been published. This makes the frame alignment statically stable over the usable regenerative region. The interested reader is referred to [2]-[4], [8].

The potential of stator resistance parameter insensitivity and absence of integration in NFO provide the motivation to investigate whether this potential can be realised in reality. Given that the static regenerative instability mentioned above has been solved, this paper will address aspects of the influence of parameter inaccuracy on the frame alignment performance of NFO. Specifically, the paper will address the effects of stator resistance and magnetising inductance error. These are the two parameters that determine the performance of stator flux oriented NFO. ${ }^{1}$

To assist in this study, a digital model has been developed in Saber ${ }^{\circledR}$ that represents, in great detail, our experimental system. The block diagram for the control implemented in the system appears in Figure 3. Note the similarity with the block diagram of the stator flux oriented control of an induction machine using a current-controlled PWM inverter as described in [9]. The block "Stator Flux Estimator" includes both NFO and SFO algorithm options, which are explained in detail in [2].

The remainder of this paper is organised as follows. Section II will develop analytical expressions to quantify the effects of stator resistance and magnetising inductance errors on the frame alignment of NFO. Section III will investigate the effects of the same parameters on SFO so that a comparison of the two algorithms can be made. Each of these sections will present simulation and experimental results to verify the predicted performance. Finally Section IV will present the conclusions that can be drawn from this work.

\section{The NFO PARAMETER SEnsitivity}

The key to understanding the parameter sensitivity mechanisms of the NFO algorithm is to realise that it uses frame

\footnotetext{
${ }^{1}$ There is also a rotor oriented version of NFO that, in addition to a dependency on $L_{m}$ and $R_{s}$ knowledge, also requires knowledge of the machine leakage inductances.
} 


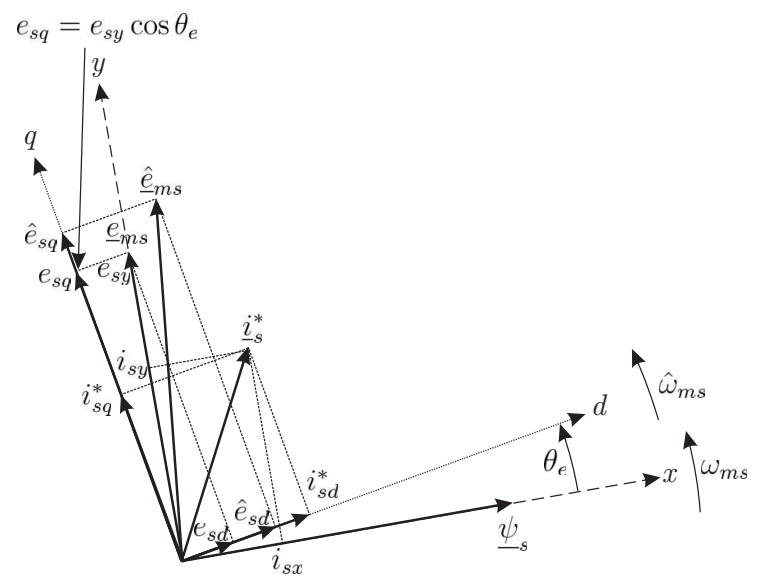

(a) Assumed $R_{s}<$ true $R_{s}$

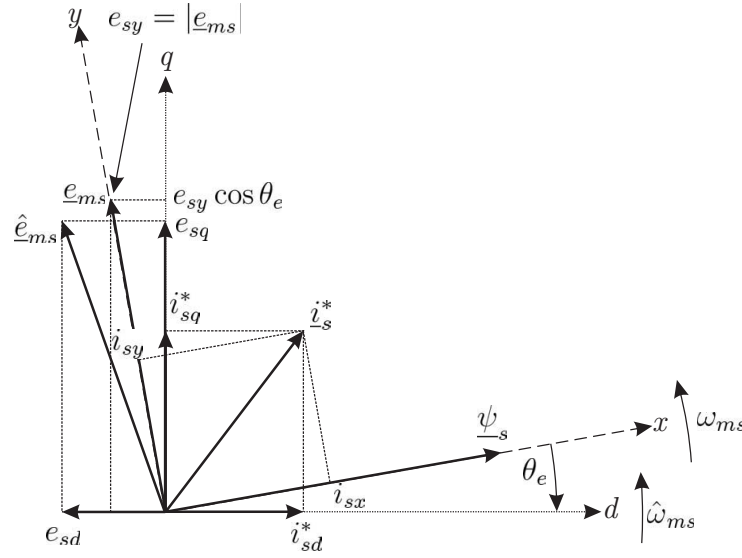

(b) Assumed $R_{s}>$ true $R_{s}$

Fig. 4: NFO reaction to stator resistance errors

misalignment to compensate for the parameter errors. If the resulting static misalignment is small, the control algorithm continues to provide approximately correct speed and torque values, even though internally it uses variables that have angular displacements. When the static misalignment reaches a critical value, an additional small disturbance makes NFO lose control over the frame position. This defines the limits of the parameter tolerance for the NFO algorithm.

\section{A. Sensitivity to the stator resistance error}

Tolerance to inaccuracy in the $R_{s}$ value can be explained using Figures $4 \mathrm{a}$ and $4 \mathrm{~b}$. First consider the situation of precisely known parameter values and perfect frame alignment in steady state. This implies that:

$$
u_{d}-R_{s} i_{s d}^{*}=e_{s d}=0 \quad u_{q}-R_{s} i_{s q}^{*}=e_{s q}=\left|\underline{e}_{m s}\right|
$$

Now let the assumed stator resistance value be smaller than the true value, i.e. $\hat{R}_{s}=R_{s}-\Delta R_{s}$. This is equivalent to adding a vector with relative coordinates $\left(\Delta R_{s} i_{s d}^{*}, \Delta R_{s} i_{s q}^{*}\right)$ to the assumed flux voltage vector. To let $e_{s d}$ become positive the control frame should turn counter-clockwise with respect to the true frame as shown in Figure 4a. In the new misaligned position

$$
\hat{e}_{s d}=e_{s y} \sin \theta_{e}+\Delta R_{s} i_{s d}^{*} \quad \hat{e}_{s q}=e_{s y} \cos \theta_{e}+\Delta R_{s} i_{s q}^{*}
$$

and the estimated angular velocity of the stator flux vector (the velocity of the control frame), according to the basic NFO algorithm [5]-[7], is:

$$
\hat{\omega}_{m s}=\frac{\hat{e}_{s q}}{\left|\underline{\psi}_{s}^{*}\right|}=\frac{e_{s y} \cos \theta_{e}+\Delta R_{s} i_{s q}^{*}}{L_{m} i_{s d}^{*}}
$$

The true angular velocity of the stator flux vector would change because of the changed flux:

$$
\omega_{m s}=\frac{e_{s y}}{\left|\underline{\psi}_{s}\right|}=\frac{e_{s y}}{L_{m}\left(i_{s d}^{*} \cos \theta_{e}-i_{s q}^{*} \sin \theta_{e}\right)}
$$

If the two angular velocities are equal, the misalignment remains statically stable. This corresponds to:

$$
\frac{e_{s y} \sin \theta_{e}}{\Delta R_{s} i_{s q}^{*}}=\frac{i_{s d}^{*} \cos \theta_{e}-i_{s q}^{*} \sin \theta_{e}}{i_{s q}^{*} \cos \theta_{e}+i_{s d}^{*} \sin \theta_{e}}=\frac{i_{s x}}{i_{s y}}
$$

Figure $4 \mathrm{a}$ shows the position of the frames corresponding to the above condition. Applying similar logic to the case when $\hat{R}_{s}=R_{s}+\Delta R_{s}$ leads to the static misalignment of Figure $4 \mathrm{~b}$. Expression (8) can be used for this case, the only difference being that $\theta_{e}$ is negative and $-\Delta R_{s}$ is used instead of $\Delta R_{s}$.

It can be easily shown that for a wide range of $\Delta R_{s}$ values of both signs and a wide range of practical ratios for $x=$ $i_{s q}^{*} / i_{s d}^{*}$, an angle value that satisfies (8) exists. Furthermore, in most cases this angle has a low sensitivity with respect to $\Delta R_{s}$, which can be approximately found by solving the equation:

$$
L_{m} \omega_{m s} \theta_{e}^{2}+\left(L_{m} \omega_{m s}+\Delta R_{s} x\right) x \theta_{e}-\Delta R_{s} x=0
$$

Equation (9) is derived from (8) by assuming $\cos \theta_{e} \approx 1$, $\sin \theta_{e} \approx \theta_{e}$ and $e_{s y} / i_{s d}^{*}=L_{m} \omega_{m s}$. It follows from (9) that the frame misalignment resulting from the stator resistance error is dependent not only on torque (through the $x$ parameter) but also on frame angular velocity. This is well known from conventional SFO algorithms. Analysis of this equation and its solutions under different conditions show that for the slow speed range and moderate to high $x$ values, the angular error can become greater than $10^{\circ}$, which is undesirable.

The sensitivity of frame alignment with respect to $\Delta R_{s}, x$, and $\omega_{m s}$ is substantially reduced when an augmented version of NFO is used as presented in [2]. In brief, this augmentation implies is that a feedback based on the $\hat{e}_{s d}$ value is added to the frame angular velocity estimation expression as follows:

$$
\hat{\omega}_{m s}=\frac{\hat{e}_{s q}-k \operatorname{sign}\left(\hat{e}_{s q}\right) \hat{e}_{s d}}{\left|\underline{\psi}_{s}^{*}\right|}
$$

With $k=0$ the expression (10) reduces to the no $e_{s d}$ feedback expression of (6). It can be further shown that if $k$ in (10) is set equal to $x$ then frame misalignment related to the stator resistance error is completely eliminated. This is obvious from the following development of the expression (10) using (5):

$\hat{\omega}_{m s}=\frac{e_{s q}+\Delta R_{s} i_{s q}^{*}-\frac{i_{s q}^{*}}{i_{s d}^{*}}\left(e_{s d}+\Delta R_{s} i_{s d}^{*}\right)}{\left|\underline{\psi}_{s}^{*}\right|}=\frac{e_{s q}-\frac{i_{s q}^{*}}{i_{s d}^{*}} e_{s d}}{\left|\underline{\psi}_{s}^{*}\right|}$

Remark 5: The $k=x$ gain selection, whilst eliminating the effects of $\Delta R_{s}$ at all operational speeds (including the 


\begin{tabular}{|c|c|c|c|c|c|c|}
\hline \multirow{2}{*}{$\begin{array}{c}\text { Para- } \\
\text { meter }\end{array}$} & \multicolumn{3}{|c|}{$\mathbf{k = 0}$} & \multicolumn{3}{c|}{$\mathbf{k = 1}$} \\
\cline { 2 - 7 } & $0 \%$ error & $-50 \%$ & $+50 \%$ & $0 \%$ error & $-50 \%$ & $+50 \%$ \\
\hline \hline $\mathbf{e}_{\mathbf{s d}}$ & $22.0 \mathrm{~V}$ & $23.6 \mathrm{~V}$ & $21.5 \mathrm{~V}$ & $2.7 \mathrm{~V}$ & $3.3 \mathrm{~V}$ & $2.1 \mathrm{~V}$ \\
\hline$\theta_{\mathbf{e}}$ & $12.2^{\circ}$ & $13.1^{\circ}$ & $11.9^{\circ}$ & $1.5^{\circ}$ & $1.9^{\circ}$ & $1.2^{\circ}$ \\
\hline
\end{tabular}

TABLE I: Experimental results on the NFO $R_{s}$ sensitivity at $\omega_{r}=50 \mathrm{rad} / \mathrm{sec}, \tau_{L}=4.7 \mathrm{~N} . \mathrm{m}$

problematic low speed region), may not be the optimal gain to use over the whole speed range to eliminate the frame misalignment from other causes. Therefore, a speed dependent gain scheduling technique should be used to get the best frame alignment performance over the whole operation region of the machine.

To confirm the analytically based conjecture that NFO has low sensitivity to the stator resistance error, a suite of simulation and experimental plots were obtained for the following conditions: after an initial fluxing period of $0.5 \mathrm{sec}$, followed by a step change from zero to $50 \mathrm{rad} / \mathrm{sec}$, the system is allowed to reach steady state. In steady state a small constant torque of $4.7 \mathrm{Nm}$ (the windage and friction losses torque) was applied, which corresponded to a current ratio of $x=0.16$. Experiments and simulations were performed for unaugmented $(k=0)$ and augmented $(k=1) \mathrm{NFO}$ with zero and $\pm 50 \%$ stator resistance error. For space reasons only one set of plots (that for $-50 \%$ underestimated $R_{s}$ ) are presented for each $k$, in Figs. 5 and 6 respectively. These results are similar in character to the $+50 \%$ resistance error case. Frame misalignments for all cases at steady state were calculated from the experimental data and are summarised in Table I.

It can be seen from the left half of Table I that for $k=0$ the angular error was substantial, being around $12^{\circ} \rightarrow 13^{\circ}$. Note that the above analysis does not account for the nonlinearities and leakage effects of the machine model on NFO frame alignment. As shown in [8], these effects manifest themselves in a non-zero static frame error even for the perfectly known machine parameters. The observed high angular error is partially attributed to these effects, and partially to dead-time error which is discussed later in this paper.

Remark 6: Table I shows that $\pm 50 \%$ stator resistance error makes almost no difference in NFO performance under the conditions of the experiment, this fact indicating that the other effects mentioned are dominating.

A significant reduction in frame misalignment is observed in Fig. 6 for $k=1$. The entries in the right half of Table I (for the $k=1$ case) show much less absolute angle error to those in the left half of the table for $k=0$.

Remark 7: Theoretical analysis, simulations and experimental results show that the NFO algorithm has low sensitivity to stator resistance error. When the additional stabilisation loop discussed in [2] is added to the control, this sensitivity decreases even further as seen from the right half of the Table I.

\section{B. Sensitivity to the magnetising inductance error}

Magnetising inductance error, according to (2), results in an erroneous frame speed estimation. The consequences of the $L_{m}$ error can be explained with the help of the relevant part of Fig. 4 (even though this diagram was originally drawn for

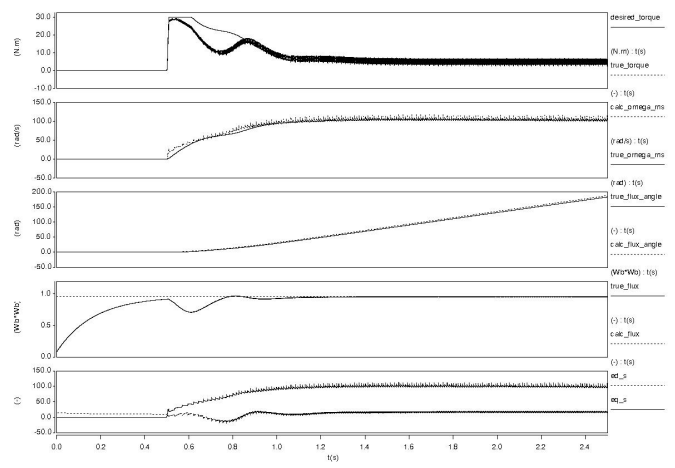

(a) Simulation data
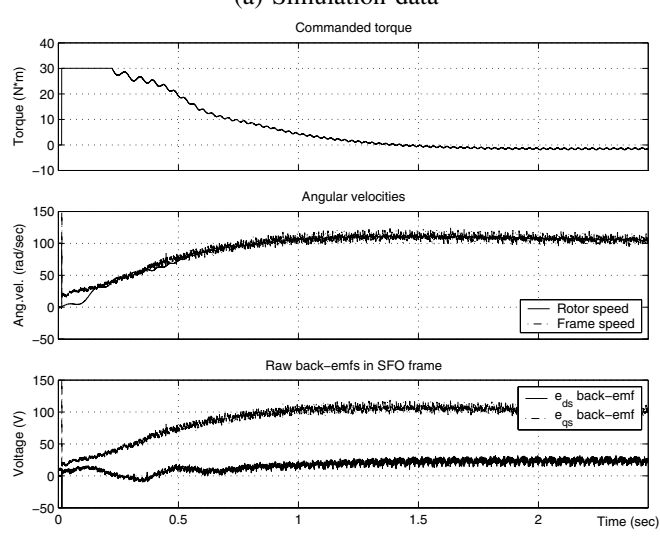

(b) Experimental data

Fig. 5: NFO start up test: $k=0, \Delta R_{s}=-50 \% R_{s}, L_{m}$ correct

the $R_{s}$ error situation). If $\hat{L}_{m}=L_{m} \pm \Delta L_{m}$, then the estimated angular velocity of the stator flux vector is

$$
\hat{\omega}_{m s}=\frac{e_{s q}}{\hat{L}_{m} i_{s d}^{*}}=\frac{e_{s y} \cos \theta_{e}}{\left(L_{m} \pm \Delta L_{m}\right) i_{s d}^{*}}
$$

which is less then or greater than its correct value, and therefore control frame is behind or ahead of the true frame. The angular velocity of the true frame is given by (7). Since the numerator of (12) decreases in magnitude as $\theta_{e}$ increases, then there is the possibility that $\theta_{e}$ will stabilise at some value. This situation will occur when the angular velocity of the estimated and true frames are the same, i.e. when:

$$
\frac{ \pm \Delta L_{m}}{L_{m}}=\sin \theta_{e}^{2}-x \sin \theta_{e} \cos \theta_{e}
$$

It can be shown that a $\theta_{e}$ that satisfies the (13) may not exist for higher $\Delta L_{m}$ values, particularly when combined with higher torques. When (13) is solvable, $\theta_{e}$ may be relatively large. The $\theta_{e}$ errors for $\Delta L m= \pm 15 \% L_{m}$ were experimentally found to be the limits for the $L_{m}$ error tolerance of the unaugmented NFO algorithm.

Plots in Fig. 7 illustrate the limiting case of $\Delta L_{m}=$ $-15 \% L_{m}$ and $k=0$. Note that when steady state is reached the $e_{s d}$ projection of the stator flux voltage (that should be zero under perfect alignment) almost reaches the value of $e_{s q}$. It should be noted that the influence of the non-linear, leakage and dead-time effects result in angular misalignment worse than predicted. The situation dramatically improves when, under the same experimental conditions, the augmented 


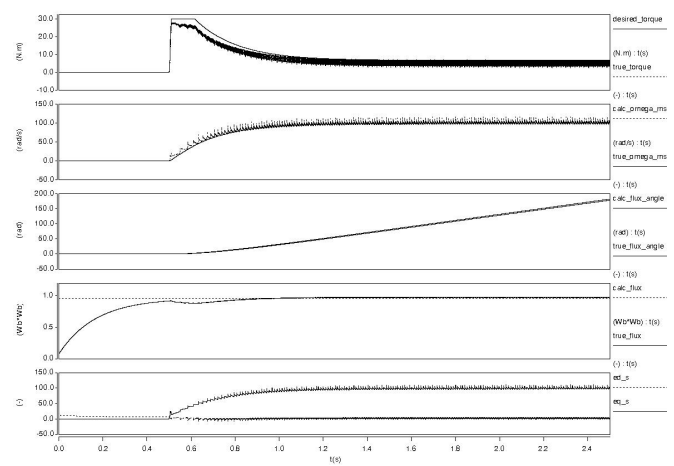

(a) Simulation data
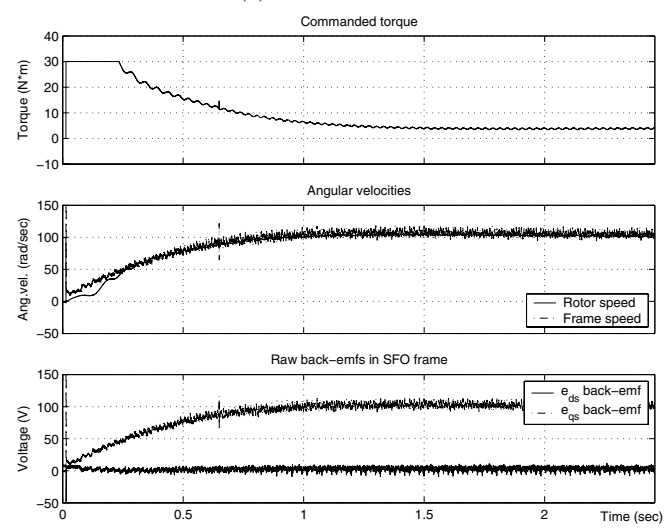

(b) Experimental data

Fig. 6: NFO start up test: $k=1, \Delta R_{s}=-50 \% R_{s}, L_{m}$ correct

\begin{tabular}{|c|c|c|c|c|c|}
\hline \multirow{2}{*}{$\begin{array}{c}\text { Para- } \\
\text { meter }\end{array}$} & \multicolumn{2}{|c|}{$\mathbf{k = 0}$} & \multicolumn{3}{c|}{$\mathbf{k}=\mathbf{1}$} \\
\cline { 2 - 6 } & $0 \%$ error & $-15 \%$ & $0 \%$ error & $-15 \%$ & $+15 \%$ \\
\hline \hline $\mathbf{e}_{\mathbf{s d}}$ & $22.0 \mathrm{~V}$ & $89.5 \mathrm{~V}$ & $2.7 \mathrm{~V}$ & $15.1 \mathrm{~V}$ & $-10.5 \mathrm{~V}$ \\
\hline$\theta_{\mathbf{e}}$ & $12.2^{\circ}$ & $43.4^{\circ}$ & $1.5^{\circ}$ & $8.7^{\circ}$ & $-6.0^{\circ}$ \\
\hline
\end{tabular}

TABLE II: Experimental results on the NFO $L_{m}$ sensitivity at $\omega_{r}=50 \mathrm{rad} / \mathrm{sec}, \tau_{L}=4.7$ N.m

version of NFO with $k=1$ is applied, as illustrated by the simulation and experimental plots of Fig. 8. The steady state angular error results, for both $k=0$ and $k=1$, are summarised in Table II.

Remark 8: To summarise, the NFO algorithm is relatively sensitive to the accurate knowledge of the magnetising inductance. However, in our experience, the accuracy of a simple no-load test was enough to get a satisfactory estimate for $L_{m}$. Based on this value, the machine can be successfully started under the NFO control. The $L_{m}$ value can be further on-line fine tuned by minimising the monitored $e_{s d}$ value at no load. Application of the stabilisation feedback, as seen from the right half of Table II, significantly improves the algorithm's tolerance to $L_{m}$ error and makes fine tuning unnecessary.

\section{Sensitivity to the dead time error}

Experimental investigation of the dead-time effect on the frame misalignment was not possible because of the fixed dead-time constraint in the experimental hardware. The PWM modulator block in Fig. 3 internally has a predictive current control loop [10], which implicitly compensates the output voltage to account for dead-time over the majority of the

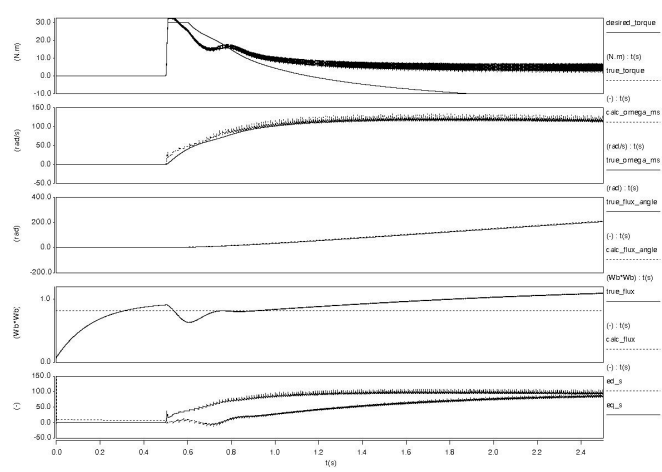

(a) Simulation data
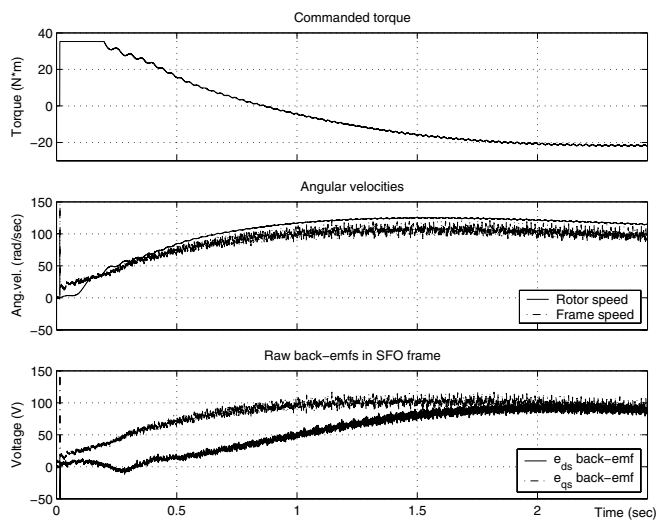

(b) Experimental data

Fig. 7: NFO start up test: $k=0, R_{s}$ correct, $\Delta L_{m}=$ $-15 \% L_{m}$

\begin{tabular}{|c|c|c|c|c|}
\hline \multirow{2}{*}{$\begin{array}{c}\text { Para- } \\
\text { meter }\end{array}$} & \multicolumn{2}{|c|}{$\mathbf{k = 0}$} & \multicolumn{2}{c|}{$\mathbf{k}=\mathbf{1}$} \\
\cline { 2 - 5 } & $0.2 \mu \mathrm{secs}$ & $4 \mu \mathrm{secs}$ & $0.2 \mu \mathrm{secs}$ & $4 \mu \mathrm{secs}$ \\
\hline \hline $\mathbf{e}_{\mathbf{s d}}$ & $3.8 \mathrm{~V}$ & $12.3 \mathrm{~V}$ & $0.6 \mathrm{~V}$ & $3.8 \mathrm{~V}$ \\
\hline$\theta_{\mathbf{e}}$ & $2.2^{\circ}$ & $7.1^{\circ}$ & $0.4^{\circ}$ & $2.2^{\circ}$ \\
\hline
\end{tabular}

TABLE III: Simulation results summary on NFO sensitivity to dead time error at $\omega_{r}=50 \mathrm{rad} / \mathrm{sec}, \tau_{L}=4.7 \mathrm{~N} . \mathrm{m}$

positive and negative current cycles [11]. However, this means that the duty cycles, $d_{\alpha}$ and $d_{\beta}$, have the dead-time error built into them. These duty cycles are used to calculate the $u_{d}$ and $u_{q}$ input voltages used in NFO.

Since dead-time results in an erroneous calculated input voltages, then an equation similar to (8) for $\Delta R_{s}$ can be obtained for $\pm \Delta u_{q}$ - the error in q-axis voltage due to the dead time in the inverter. Note that for the non-augmented NFO only the q-axis voltage error is relevant. The following expression results:

$$
\frac{ \pm \Delta u_{q}}{e_{s y}}=\frac{i_{s y}}{i_{s x}} \sin \theta_{e}=\frac{\sin \theta_{e}+x \cos \theta_{e}}{\cos \theta_{e}-x \sin \theta_{e}}
$$

The summary of the steady state frame angular errors obtained by simulations is presented in Table III for $0.2 \mu \mathrm{sec}$ and $4 \mu \mathrm{sec}$ dead-time errors, the latter corresponding to that of the experimental set up under the same speed.

Remark 9: To summarise, the NFO algorithm has low sensitivity to the dead time errors similar to its tolerance of $R_{s}$ errors. The angular misalignment may become larger at slow 


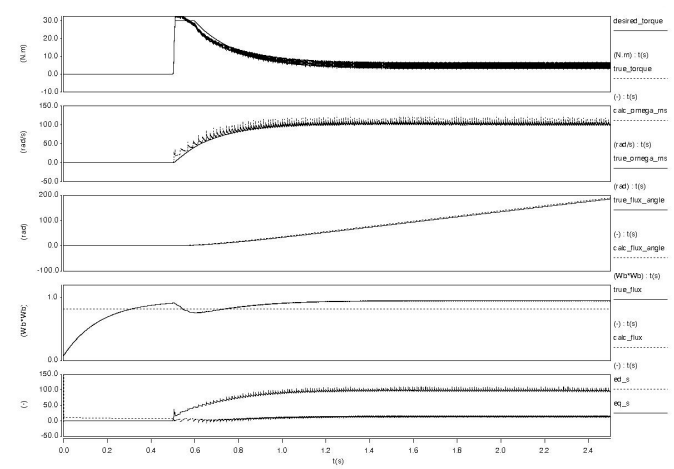

(a) Simulation data
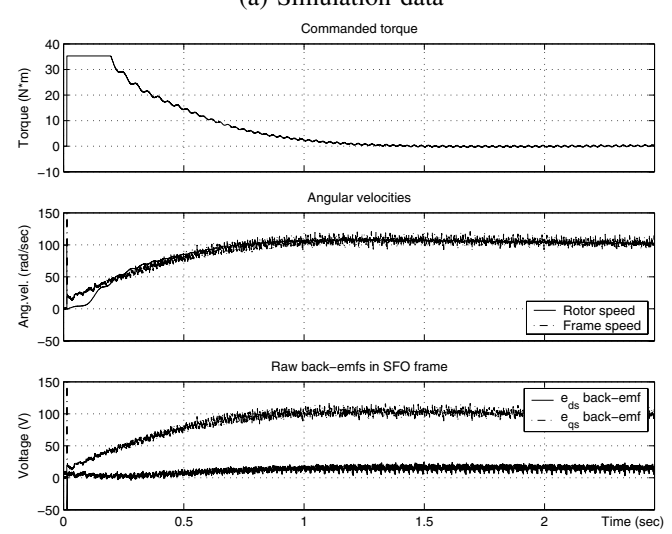

(b) Experimental data

Fig. 8: NFO start up test: $k=1, R_{s}$ correct, $\Delta L_{m}=$ $-15 \% L_{m}$

speeds due to the relatively large effect of dead-time here. Augmented NFO significantly reduces such misalignment.

\section{COMPARISON to THE SFO PARAMETER SENSITIVITy}

NFO's natural self alignment, which is explained in [2], is inherited from the traditional SFO. However, there are small but important differences in how frame alignment is achieved in NFO and SFO.

Assuming SFO control, consider initially that there is alignment between the control frame and the stator flux vector positions followed by a disturbance $\theta_{e}$. The true and the estimated angular velocities are respectively:

$$
\omega_{m s}=\frac{e_{s y}}{L_{m}\left(i_{s d}^{*} \cos \theta_{e}-i_{s q}^{*} \sin \theta_{e}\right)}
$$

and

$$
\hat{\omega}_{m s}=\frac{e_{s y} \cos \theta_{e}}{L_{m} i_{s d}^{*}+\int e_{s y} \sin \theta_{e} d t}
$$

where $\left|\underline{\psi}_{s}\right|\left(t_{0}\right)=L_{m} i_{s d}^{*}-$ i.e the initial condition.

It can be seen from the above formulae that there cannot be static misalignment between the two frames, otherwise the term in the denominator of (16) will integrate up or down without limit. The two velocities can only be equal when $\theta_{e}=0$ but at this point the frames always have a relative acceleration. This can be shown by taking a derivative of the relative velocity at $\theta_{e}=0$. As a result, the selfalignment property occurs in an oscillatory manner. This is consistent with observations made in [9], that state that stator flux estimation based on pure $e_{s d}$ integration results in a marginally stable system. The simulation and experimental results of Fig. 10 further support this conclusion.

In a simulation of start-up under SFO control (Fig.10a) undamped oscillations at the frequency $\omega_{m s}$ are observed in the calculated speed and flux. Note that there are no oscillations in the actual machine speed and flux. However, as the estimated (oscillatory) angular position is used to provide the commanded torque current, a component of frequency $\omega_{m s}$ is present in the machine torque (this is low pass filtered by the load dynamics and therefore minimises any effect on machine speed). In the corresponding experiment, to be able to start the machine under the SFO control, we had to explicitly limit the output of the flux integrator. Much higher oscillations than those in the simulation are seen in the experimental plots of Fig. 10b.

\section{A. Linearised stability analysis}

The following linearised analysis assists in understanding of the stability issues of the SFO technique. First consider the original (unaugmented) SFO algorithm with a pure integrator for flux estimation, under the steady state operation.

Assume that the angular position of the stator flux vector in steady state is: $\theta_{m s}(t)=\omega_{m s} t$. An error of $\theta_{e}$ may occur in the estimated frame position, so that $\hat{\theta}_{m s}(t)=\omega_{m s} t+\theta_{e}$. The stator flux voltage vector in steady state is $90^{\circ}$ ahead of the flux vector, i.e. $\underline{e}_{m s}=e_{m s} e^{j\left(\omega_{m s} t+90^{\circ}\right)}$. Considering Fig. 1, it is clear that the SFO algorithm implies the following transformations of this vector. First the stator flux voltage vector is converted to the stator flux frame:

$$
\underline{e}_{m s \hat{\psi}_{s}}=\underline{e}_{m s} e^{-j \hat{\theta}_{m s}(t)}=e_{m s} e^{j\left(90^{\circ}-\theta_{e}\right)}
$$

The projections of this vector onto the control frame axes are:

$$
e_{s d}=e_{m s} \sin \left(\theta_{e}\right) \approx e_{m s} \theta_{e} \quad e_{s q}=e_{m s} \cos \left(\theta_{e}\right) \approx e_{m s}
$$

The $e_{s d}$ projection is then integrated to get estimated flux:

$$
\hat{\psi}_{s}=\psi_{s}^{*}+e_{m s} \int \theta_{e} d t
$$

The angular velocity of the control frame is estimated as:

$$
\hat{\omega}_{m s}=\frac{e_{s q}}{\hat{\psi}_{s}}=\frac{e_{m s}}{\psi_{s}^{*}+e_{m s} \int \theta_{e} d t}=\frac{e_{m s}}{\psi_{s}^{*}\left(1+\frac{e_{m s}}{\psi_{s}^{*}} \int \theta_{e} d t\right)}
$$

Using the approximation $(1+z)^{-1} \approx 1-z$ for $z<<1$ one can write:

$$
\hat{\omega}_{m s} \approx \frac{e_{m s}}{\psi_{s}^{*}}\left(1-\frac{e_{m s}}{\psi_{s}^{*}} \int \theta_{e} d t\right)=\omega_{m s}-\frac{e_{m s}^{2}}{\left(\psi_{s}^{*}\right)^{2}} \int \theta_{e} d t
$$

Finally, the frame angular velocity is integrated to get the angular position of the control frame:

$$
\hat{\theta}_{m s}(t)=\int \hat{\omega}_{m s} d t=\omega_{m s} t-\int\left(\frac{e_{m s}^{2}}{\left(\psi_{s}^{*}\right)^{2}} \int \theta_{e} d t\right) d t
$$

Based on the above expressions, a linearised frame angle disturbance model can be developed as shown in Fig. 9a. The input to the model is an angular position disturbance $\Delta \theta_{e}$ that integrates into a zero mean value over a period of time. If a non-zero mean $\Delta \theta_{e}$ occurs, then (16) predicts that 


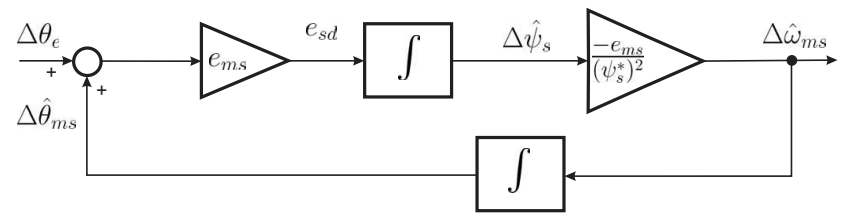

(a) Unaugmented SFO, $k=0$

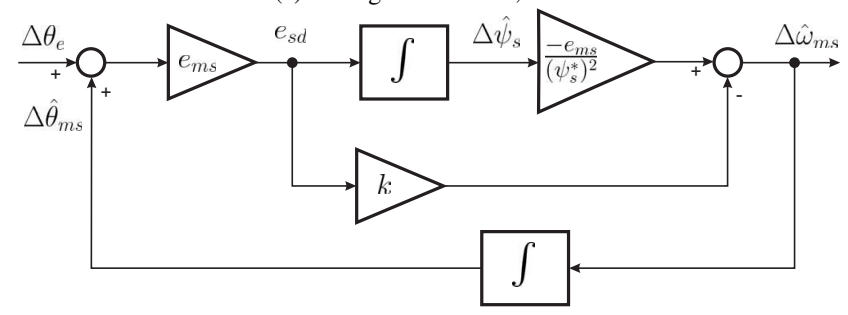

(b) Augmented SFO, $k=1$

Fig. 9: Linearised frame angular position disturbance model for the SFO algorithm.

the estimated frame angular velocity and hence control frame position changes to eliminate the disturbance. From Fig. 9a the transfer function of the angular velocity estimation error $\left(\Delta \omega_{m s}\right)$ due to the disturbance $\Delta \theta_{e}$ is:

$$
\frac{\Delta \Omega_{m s}(s)}{\Delta \Theta_{e}(s)}=-\frac{e_{m s}^{2}}{\left(\psi_{s}^{*}\right)^{2}} \frac{s}{s^{2}+\frac{e_{m s}^{2}}{\left(\psi_{s}^{*}\right)^{2}}}=-\omega_{m s}^{2} \frac{s}{s^{2}+\omega_{m s}^{2}}
$$

This transfer function has one zero at the origin and two poles $p_{1,2}= \pm j \omega_{m s}$ on the imaginary axis, corresponding to observed undamped oscillations at the natural frequency $\omega_{m s}$.

\section{B. Effect of the stabilisation loop}

Now consider the augmented version of the SFO algorithm with $e_{s d}$ feedback and a gain $k$ similar to augmented NFO. Applying the same logic as for unaugmented SFO, the linearised disturbance model block diagram of Fig. 9b can be developed. The following transfer function can be determined from the diagram:

$$
\frac{\Delta \Omega_{m s}(s)}{\Delta \Theta_{e}(s)}=-\frac{\omega_{m s} \psi_{s}^{*} k s^{2}+\omega_{m s}^{2} s}{s^{2}+\omega_{m s} \psi_{s}^{*} k s+\omega_{m s}^{2}}
$$

with zeros $z_{1}=0, z_{2}=-\omega_{m s} /\left(k \psi_{s}^{*}\right)$ and poles given by:

$$
p_{1,2}=-\omega_{m s}\left(k \psi_{s}^{*} / 2\right) \pm \sqrt{\left(k \psi_{s}^{*} / 2\right)^{2}-1}
$$

It is clear that with the augmentation applied to SFO, the $k$ parameter controls the damping ratio. For $k \psi_{s}^{*}<2$ the system will react to a short pulse disturbance with damped oscillations with a damping ratio of $k \psi_{s}^{*} / 2$. For higher $k$ the reaction to the disturbance will be aperiodic. Using very high $k$ values is undesirable as one of the poles would move close to the origin.

The effect of the augmentation on the SFO performance can be in seen by comparing the simulation and experimental results for the $k=0$ and $k=1$ cases shown in Figs. 10 and 11 respectively. As the result of the augmentation, the oscillations of the angular velocity, flux and torque are no longer present in Fig. 11.

Remark 10: The augmentation of the SFO algorithm with $e_{s d}$ feedback damps the $\omega_{m s}$ frequency oscillations present in SFO implemented with pure integration to estimate flux.

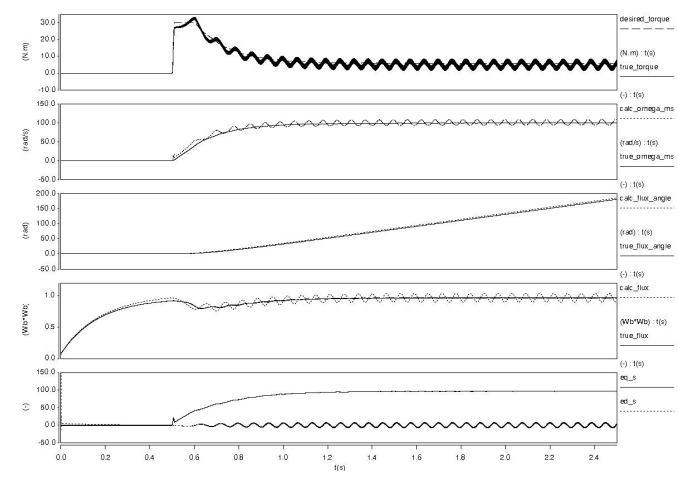

(a) Simulation data
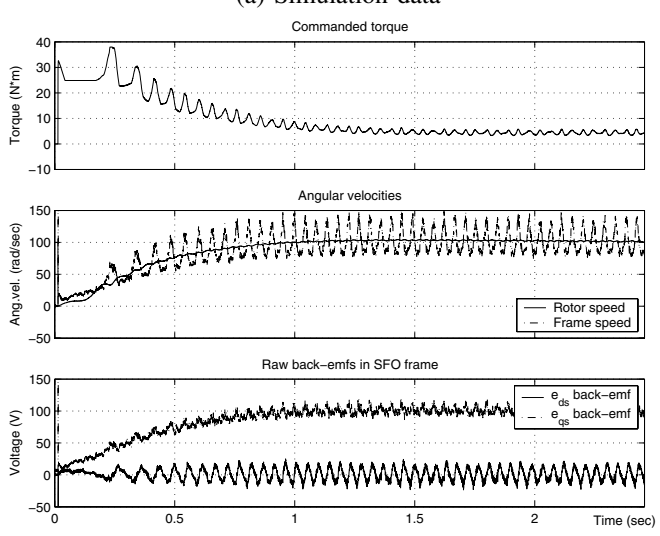

(b) Experimental data

Fig. 10: SFO test: $k=0, R_{s}$ correct, $L_{m}$ correct

\section{Sensitivity to the parameter errors}

Compared to NFO, frame alignment in SFO is very insensitive to the $L_{m}$ error, as it only uses the magnetising inductance to calculate the set point for the torque current (see Fig. 1). The way stator resistance error influences SFO performance is also quite different from NFO. While NFO can compensate for $R_{s}$ error by adding a small static angle error to its control frame position, SFO does not have this compensation mechanism available. Hence, $R_{s}$ is critical for SFO performance and error can instigate or exacerbate the above mentioned control frame oscillations. Dead-time errors also have a similar influence on SFO.

Application of the $e_{s d}$ feedback however, results in reasonable performance for $\pm 50 \% R_{s}$ errors under the experimental conditions presented in this paper. For space reasons, only one set of results for $-50 \% R_{s}$ are presented in Fig. 12. SFO sensitivity, with and without augmentation, to the parameter errors at low speed are still to be investigated.

Remark 11: To summarise, the SFO algorithm is insensitive to errors in $L_{m}$, but sensitive to $R_{s}$ and dead-time errors. The application of the $e_{s d}$ feedback decreases SFO stator resistance sensitivity.

\section{CONCLUSIONS}

This paper has presented a thorough investigation of the effects of parameter error on the frame alignment accuracy of the NFO and SFO based control algorithms. The main conclusions from the paper are: 


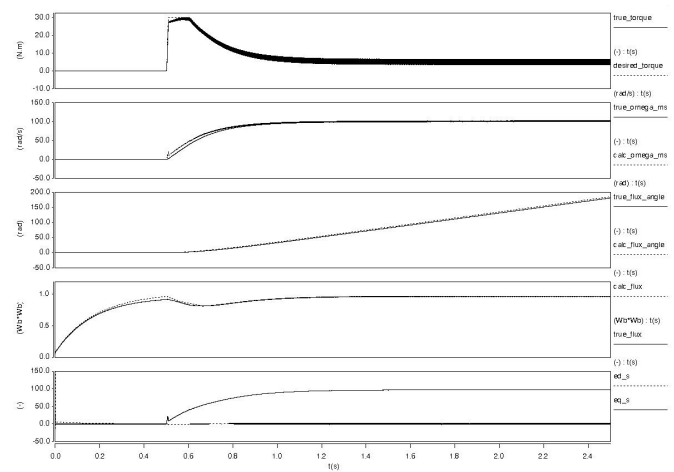

(a) Simulation data

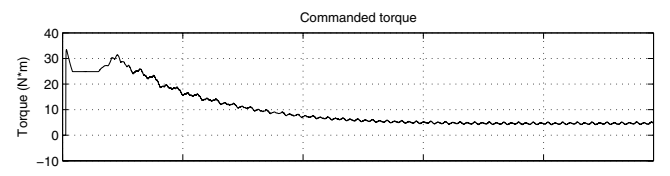

Angular velocities
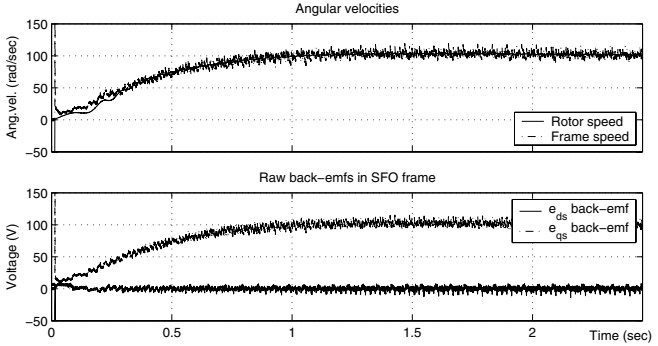

(b) Experimental data

Fig. 11: SFO test: $k=1, R_{s}$ correct, $L_{m}$ correct

- Frame alignment with NFO is intrinsically tolerant to errors in $R_{s}$ and dead-time errors.

- NFO $R_{s}$ and dead-time insensitivity can be further enhanced by the augmenting the original algorithm with $e_{s d}$ feedback. This is especially important for acceptable low speed operation.

- NFO frame alignment is more sensitive to errors in $L_{m}$, as compared to $R_{s}$. However, errors of up to $15 \%$ can be tolerated.

- Augmented NFO can tolerate larger errors in $L_{m}$ before frame alignment becomes unstable.

- SFO control systems using pure integration for flux estimation are marginally stable with respect to control frame alignment. The frame oscillation natural frequency is $\omega_{m s} \mathrm{rad} / \mathrm{sec}$.

- If an $e_{s d}$ feedback augmentation strategy is used with SFO, similar to that applied to NFO, then the frame alignment oscillations can be damped (when appropriate feedback gains are used).

- $\mathrm{SFO}$ is more sensitive to $R_{s}$ and dead-time errors relative to NFO. However, feedback augmentation of SFO decreases this sensitivity.

- Frame alignment in SFO is not sensitive to $L_{m}$ errors.

All of the above conclusions have been confirmed by analysis, simulation and experimental results. An overall conclusion is the NFO has some interesting parameter robustness properties, and should offer superior performance to SFO, especially at low speed.

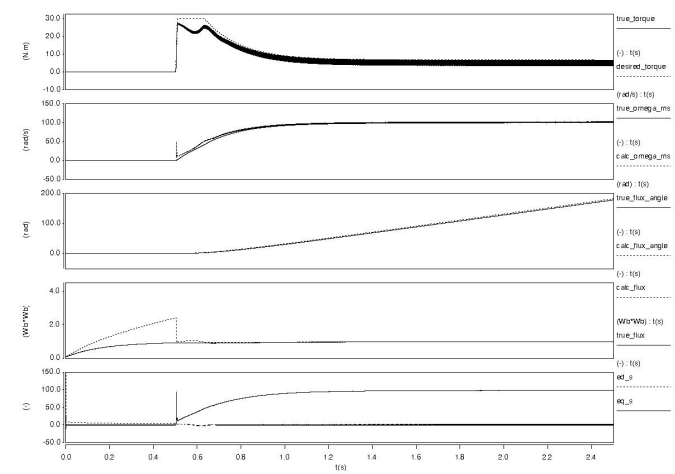

(a) Simulation data
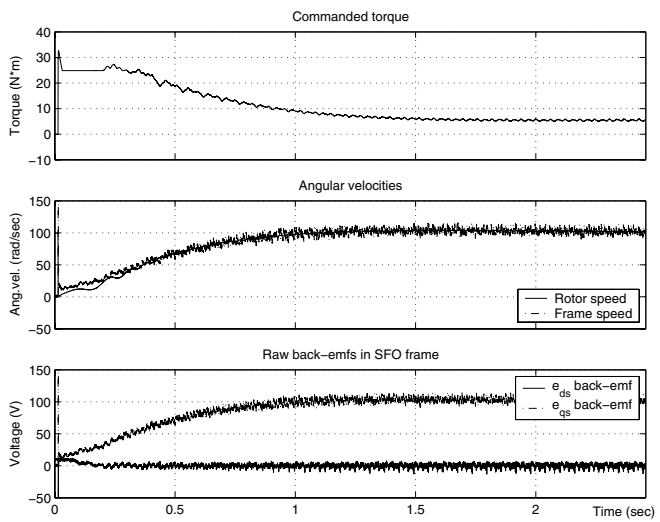

(b) Experimental data

Fig. 12: SFO test: $k=1, \Delta R_{s}=-50 \% R_{s}, L_{m}$ correct

\section{REFERENCES}

[1] B. K. Bose, Modern Power Electronics and AC Drives. Prentice-Hall, 2002.

[2] R. Betz and G. Mirzaeva, "Frame alignment stability issues in natural field orientation," in CDROM Proceedings of the IEEE IAS Annual Meeting. IEEE, October 2006.

[3] G. Mirzaeva and R. Betz, "An improved natural field orientation control of a current fed induction machine," in CDROM Proceedings of IEEEIEMDC 2007, Antalya, Turkey, May 2007.

[4] — "Natural field orientation concept: a tutorial," in CDROM Proceedings of IEEE-IEMDC 2007, Antalya, Turkey, May 2007.

[5] R. Jönsson, "Method and apparatus for controlling an AC motor," United States Patent 4,458,193, July 1984.

[6] R. Jönsson, "Method and apparatus for controlling an ac induction motor by indirect measurement of the air-gap voltage," United States Patent 5,294,876, March 1994.

[7] R. Jönsson and W. Leonhard, "Control of an induction motor without a mechanical sensor, based on the principle of natural field orientation (NFO)," in International Power Electronics Conference, IPEC'95, April 1995, pp. 1-6.

[8] G. Mirzaeva and R. Betz, "Analysis of frame alignment issues in natural field orientation including non-linear and leakage inductance effects," September 2007, accepted for EPE2007, Aalborg, Denmark.

[9] P. Vas, Sensorless Vector and Direct Torque Control. Oxford University Press, 2003.

[10] S. J. Henriksen, R. E. Betz, and B. J. Cook, "Digital hardware implementation of a current controller for IM variable-speed drives," IEEE Transactions on Industry Applications, vol. 35, no. 5, pp. 1021-1029, Sept/Oct 1999

[11] T. J. Summers and R. E. Betz, "Dead-time issues in predictive current control," IEEE Trans. Ind. Appl., vol. 40, no. 3, pp. 835-844, May/Jun. 2004. 